\title{
Single versus Multiple Level Sectioning for the Subtyping of Basal-Cell Carcinoma: A Retrospective Study
}

\author{
Lieke C.J. van Delft ${ }^{a}$ b Patty J. Nelemans ${ }^{c}$ Myrurgia Abdul Hamid ${ }^{d}$ \\ Nicole W.J. Kelleners-Smeets ${ }^{a, b}$ \\ ${ }^{a}$ Department of Dermatology, Maastricht University Medical Center+, Maastricht, The Netherlands; ${ }^{b} \mathrm{GROW}$ \\ Research Institute for Oncology and Developmental Biology, Maastricht University, Maastricht, The Netherlands; \\ 'Department of Epidemiology, Maastricht University, Maastricht, The Netherlands; ${ }^{\mathrm{d} D e p a r t m e n t}$ of Pathology, \\ Maastricht University Medical Center+, Maastricht, The Netherlands
}

\section{Keywords}

Basal-cell carcinoma $\cdot$ Punch biopsy $\cdot$ Histological subtype

\begin{abstract}
Background: The histological subtype of basal-cell carcinoma (BCC) is often based on a punch biopsy; only a small part is evaluated, possibly leading to misclassification. Consensus on the optimal approach to process punch biopsies is lacking, though accurate subtyping is important for appropriate treatment. Objective: The aim is to investigate whether evaluating 4 levels of a punch biopsy instead of 1 or 2 levels leads to more accurate subtyping of BCC. Methods: In a retrospective study we evaluated 87 punch biopsies of histologically confirmed BCCs. The primary outcome was the proportion of "more aggressive" BCCs (nonsuperficial vs. superficial, infiltrative vs. nodular subtype) that was missed by evaluation on 1 or 2 levels, using 4-level diagnosis as reference standard. Results: Eighty-five cases were available for analysis. Subtyping based on 1 level resulted in discrepancies with 4-level diagnosis in $16.5 \%$ of all cases. Underdiagnosis oc-
\end{abstract}

\begin{tabular}{ll}
\hline karger@karger.com & (C) 2019 The Author(s) \\
www.karger.com/drm & Published by S. Karger AG, Basel Karger \\
& This article is licensed under the Creative Commons Attribution- \\
Karger & NonCommercial-NoDerivatives 4.0 International License (CC BY- \\
NC-ND) (http://www.karger.com/Services/OpenAccessLicense). \\
Usage and distribution for commercial purposes as well as any dis- \\
tribution of modified material requires written permission.
\end{tabular}

curred in 14 of 58 nonsuperficial BCCs $(24.1 \%, 95 \%$ Cl: $13.9-$ 37.2). Seven of 38 nodular BCCs (18.4\%, 95\% Cl: 7.74-34.3) were diagnosed as superficial in 1 level, and 7 of 20 infiltrative BCCs (35\%, 95\% Cl: 15.4-59.2) were diagnosed as superficial $(n=2)$ or nodular $(n=5)$ in 1 level. Conclusion: In order to maximize correct subtyping and plan appropriate treatment, we advise to evaluate at least 2 , but preferably more, levels of a punch biopsy to determine the BCC subtype.

(c) 2019 The Author(s)
Published by S. Karger AG, Basel

\section{Introduction}

Diagnosis and subtype of basal-cell carcinoma (BCC) are often based on the histological evaluation of a 3- to 4-mm punch biopsy which is important to determine the appropriate treatment [1]. A review of the literature showed that a "more" aggressive subtype (nodular vs. superficial and infiltrative vs. superficial/nodular subtypes) was missed in biopsy material in approximately $15 \%$ of the BCCs [2]. Attempts to improve the histological evalu- 
ation of skin biopsy material showed that deeper sectioning is important to specify diagnoses [3, 4]. Up until now, there has been no universal protocol on how to section biopsy material of clinically suspect BCCs [3]. Therefore, this retrospective study aims to assess how often evaluation of histological sections at 1 or 2 levels instead of 4 levels fails to detect the "more" aggressive component of a BCC.

\section{Methods}

For further details, see the online supplementary material (see www.karger.com/doi/10.1159/000503320 for all online suppl. material) (Fig. 1) [5].

\section{Results}

We analyzed 85 histologically confirmed primary BCCs; 4 levels of the punch biopsy showed 27 superficial (31.8\%), 38 nodular (44.7\%) and 20 infiltrative (23.5\%) BCCs. The histological subtype of a BCC based on 1 versus 4 levels was discordant in 16.5\% (14/85, 95\% CI: 9.3$26.1 \%$ ) of the cases (Table 1, Fig. 2). Six biopsies (number needed to treat) need to be sectioned at 4 levels instead of 1 level to prevent $1 \mathrm{BCC}$ from being underdiagnosed.

Of the 58 nonsuperficial (nodular and infiltrative) subtypes, 7 of 38 nodular BCCs (18.4\%, 95\% CI: 7.74-34.3) were diagnosed as superficial in 1 level, and 7 of 20 infiltrative BCCs (35\%, 95\% CI: 15.4-59.2) were diagnosed as superficial $(n=2)$ or nodular $(n=5)$ in 1 level. Thus, underdiagnosis occurred in $24.1 \%$ (95\% CI: $13.9-37.2 \%$ ) of the 58 nonsuperficial subtypes. Evaluation of 2 levels led to a better identification of aggressive components. When subtyping of BCC was based on 2 levels, underdiagnosis occurred in $12.1 \%$ (7/58, 95\% CI: $5.0-23.3 \%)$ in levels 1 and $4(p=0.016), 6.9 \%(4 / 58,95 \%$ CI: $1.9-16.7 \%)$ in levels 2 and $4(p=0.031)$ and $8.6 \%(5 / 58,95 \%$ CI: $2.8-19.0 \%)$ in levels 3 and $4(p=0.031)$ (Table 1). The difference in proportion of missed nodular or infiltrative subtypes between 1-level and 2-level evaluation was statistically significant.

\section{Discussion and Conclusion}

It is prudent to distinguish purely superficial from nonsuperficial (and thus "more" aggressive) BCCs and nodular from infiltrative BCCs in order to determine the

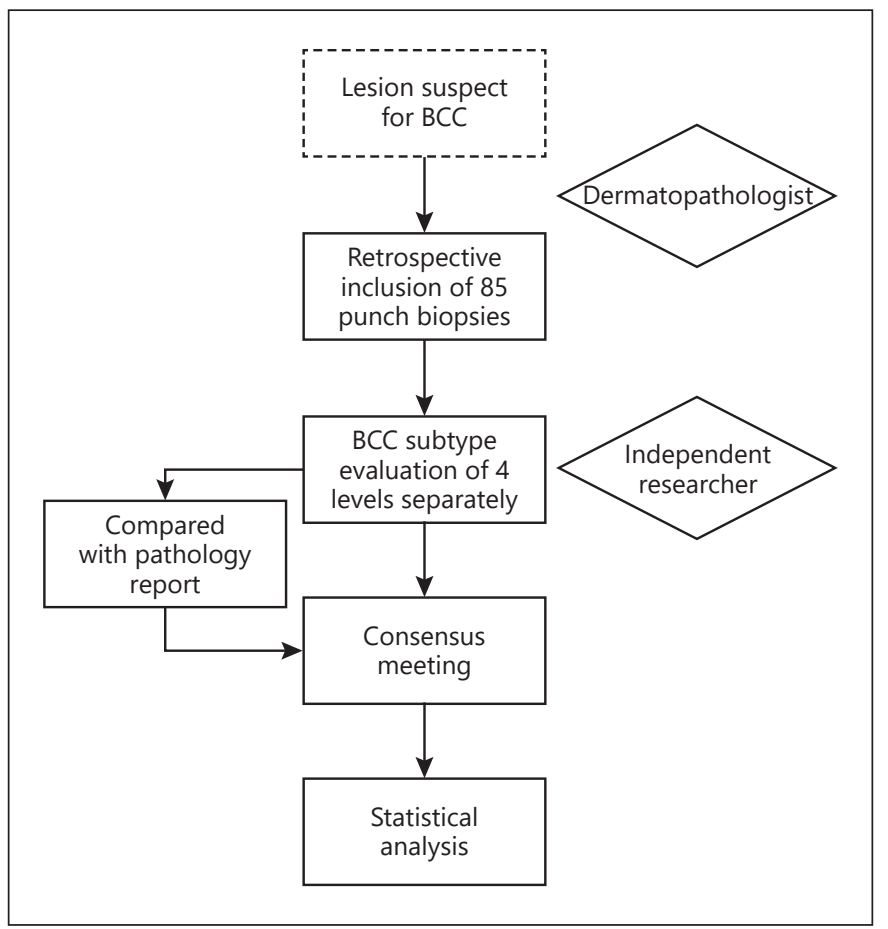

Fig. 1. Flowchart of the Methods. BCC, basal-cell carcinoma.

appropriate therapy [1]. If subtyping is based on 1 level only, approximately 1 out of $6(16.5 \%)$ BCCs is potentially incorrectly subtyped and consequently undertreated. Six biopsies (number needed to treat) need to be sectioned at 4 levels instead of 1 level to prevent 1 BCC from being underdiagnosed. Evaluation of 2 levels led to significantly less misdiagnosis of aggressive components varying from 7.0 to $12.0 \%$.

Two previous studies describe deeper sectioning techniques for optimization of BCC subtyping based on biopsy material $[3,6]$. Mudaliar et al. [3] aimed to investigate the rate and utility of retrospective deeper sectioning of 1 level compared to prospective sectioning of 2 levels of punch biopsies suspect for BCC. For diagnosing BCCs, evaluation on 1 level was often sufficient, though deeper sectioning was valuable for subtyping [3]. Nguyen et al. [6] evaluated an extensive step sectioning technique for punch biopsies suspect for BCC. Biopsy material was sectioned up to 5 levels; of each level a ribbon of 10 samples was sectioned of which the central 2 were evaluated. Using this technique, they detected 22.4\% additional "more" aggressive subtype BCCs compared to evaluating 1 level [6]. This protocol, however, seems complex, and possibly time-consuming and labor-intensive. 
Fig. 2. Histological subtype of basal-cell carcinoma (BCC) based on 1 or 2 levels versus 4 levels of a punch biopsy. Figures above the columns are percentages.

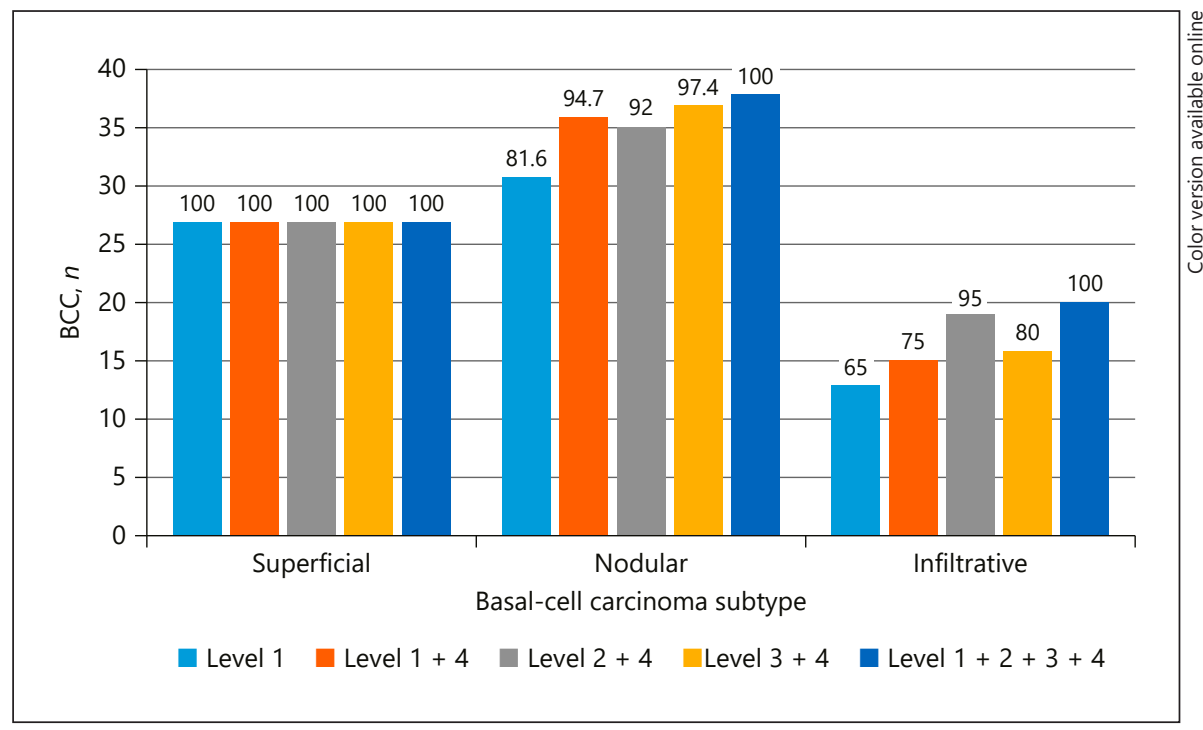

Table 1. Histological subtype of basal-cell carcinoma based on 1 or 2 levels versus 4 levels of a punch biopsy

\begin{tabular}{|c|c|c|c|c|c|}
\hline & \multicolumn{5}{|c|}{ Four-level evaluation } \\
\hline & no tumor & superficial BCC & nodular BCC & infiltrative BCC & total \\
\hline \multicolumn{6}{|c|}{ One-level evaluation (level 4) } \\
\hline No tumor & $0(0)$ & $0(0)$ & $1(2.6)$ & $0(0)$ & 1 \\
\hline Superficial BCC & - & $27(100)$ & $6(15.8)$ & $2(10.0)$ & 35 \\
\hline Nodular BCC & - & - & $31(81.6)$ & $5(25.0)$ & 36 \\
\hline Infiltrative BCC & - & - & - & $13(65.0)$ & 13 \\
\hline Total & $0(0)$ & 27 & 38 & 20 & 85 \\
\hline \multicolumn{6}{|c|}{ Two-level evaluation (levels $1+4$ ) } \\
\hline No tumor & $0(0)$ & $0(0)$ & $1(2.6)$ & $0(0)$ & 1 \\
\hline Superficial BCC & - & $27(100)$ & $1(2.6)$ & $0(0)$ & 28 \\
\hline Nodular BCC & - & - & $36(94.7)$ & $5(25.0)$ & 41 \\
\hline Infiltrative BCC & - & - & - & $15(75.0)$ & 15 \\
\hline Total & $0(0)$ & 27 & 38 & 20 & 85 \\
\hline \multicolumn{6}{|c|}{ Two-level evaluation (levels $2+4)$} \\
\hline No tumor & $0(0)$ & $0(0)$ & $1(2.6)$ & $0(0)$ & 1 \\
\hline Superficial BCC & - & $27(100)$ & $2(5.3)$ & $0(0)$ & 29 \\
\hline Nodular BCC & - & - & $35(92)$ & $1(5.0)$ & 36 \\
\hline Infiltrative BCC & - & - & - & $19(95.0)$ & 19 \\
\hline Total & $0(0)$ & 27 & 38 & 20 & 85 \\
\hline \multicolumn{6}{|c|}{ Two-level evaluation (levels $3+4$ ) } \\
\hline No tumor & $0(0)$ & $0(0)$ & $0(0)$ & $0(0)$ & 0 \\
\hline Superficial BCC & - & $27(100)$ & $1(2.6)$ & $1(5.0)$ & 29 \\
\hline Nodular BCC & - & - & $37(97.4)$ & $3(15.0)$ & 40 \\
\hline Infiltrative BCC & - & - & - & $16(80.0)$ & 16 \\
\hline Total & $0(0)$ & 27 & 38 & 20 & 85 \\
\hline
\end{tabular}

Data are given as $n(\%)$. Italicized figures indicate concordant pairs. BCC, basal-cell carcinoma. 
The protocol for the processing of BCC punch biopsy material differs between pathology laboratories; this difference in trimming strategies is however not the only factor of influence that may cause sampling error of punch biopsies. Other factors contributing to the accuracy of subtyping are the size and depth of the biopsy (shave vs. punch biopsy) and the experience of the physician taking the biopsy; a biopsy should be taken of the clinically most suspect part of the BCC, which is often the thickest part [7].

A reason for evaluating only 1 level is that this could potentially reduce costs by a decreased turnaround time and smaller storage uptake. However, an erroneously treated misclassified BCC can recur, leading to extra costs $[8,9]$. The (in)convenience of deeper sectioning should be weighed against the possibility of recurrence and retreatment. Ultimately, the quantity of sectioned levels should be based on the amount of misclassified BCCs one is willing to accept.
In conclusion, in order to optimize subtyping and prevent consequential incorrect treatment and recurrence, we advise to evaluate at least $2(300-\mu \mathrm{m}$ intervals), and preferably more, levels of a punch biopsy to determine the BCC subtype.

\section{Key Message}

Basal-cell carcinoma subtypes should be evaluated on 2 or more levels of a punch biopsy.

\section{Statement of Ethics}

The institutional review board of the Maastricht University Medical Center+ in the Netherlands (METC azM/UM) approved the study protocol.

\section{Disclosure Statement}

The authors have no conflicts of interest to declare.

\section{References}

1 Trakatelli M, Morton C, Nagore E, Ulrich C, Del Marmol V, Peris K, et al.; BCC subcommittee of the Guidelines Committee of the European Dermatology Forum. Update of the European guidelines for basal cell carcinoma management. Eur J Dermatol. 2014 May-Jun; 24(3):312-29.

2 Kadouch DJ, van Haersma de With A, Limpens J, van der Wal AC, Wolkerstorfer A, Bekkenk $\mathrm{MW}$, et al. Is a punch biopsy reliable in subtyping basal cell carcinoma? A systematic review. Br J Dermatol. 2016 Aug; 175(2):401-3.

3 Mudaliar K, Speiser J, Hutchens KA. Changing Dermatopathology Protocols for Processing Small Skin Biopsies: Decreases in Slide
Quantity Does Not Affect Patient Care and Allows for Optimal Efficiency. Am J Dermatopathol. 2016 Jun;38(6):409-12.

4 Maingi CP, Helm KF. Utility of deeper sections and special stains for dermatopathology specimens. J Cutan Pathol. 1998 Mar;25(3):171-5.

5 Rippey JJ. Why classify basal cell carcinomas? Histopathology. 1998 May;32(5):393-8.

6 Nguyen KP, et al. Standard step sectioning of skin biopsy specimens diagnosed as superficial basal cell carcinoma frequently yields deeper and more aggressive subtypes. J Am Acad Dermatol. 2017;76(2):351-3. e3.

7 Cameron MC, et al. Basal cell carcinoma. II. Contemporary approaches to diagnosis, treatment, and prevention. J Am Acad Dermatol. 2019 Feb;80(2):321-39.

8 Stuart LN, Rodriguez AS, Gardner JM, Foster TE, MacKelfresh J, Parker DC, et al. Utility of additional tissue sections in dermatopathology: diagnostic, clinical and financial implications. J Cutan Pathol. 2014 Feb; 41(2):81-7.

9 Arits AH, Spoorenberg E, Mosterd K, Nelemans P, Kelleners-Smeets NW, Essers BA. Cost-effectiveness of topical imiquimod and fluorouracil vs. photodynamic therapy for treatment of superficial basal-cell carcinoma. Br J Dermatol. 2014 Dec;171(6): 1501-7. 